\title{
Removal of chromium(VI) from solution by coconut husk and palm pressed fibres
}

\begin{abstract}
Removal of chromium(VI) from aqueous solutions by coconut husk fibres (CHF) and palm pressed fibres (PPF) was investigated using batch and column techniques. Batch tests showed that $\mathrm{pH}$ range for effective $\mathrm{Cr}(\mathrm{VI})$ removal was between 1.5 and 5 for $\mathrm{CHF}$ and between 1.5 and 3 for PPF. The adsorption capacities of CHF and PPF were estimated to be 29 and $14 \mathrm{mg}$ $\mathrm{Cr}$ g-1 substrate at $\mathrm{pH}$ 2.0. In column studies, breakthrough curves of various columns containing PPF were obtained for various flow rates and bed-depths and the results were compared with Hutchin's model for adsorption column (1). The results of breakthrough curves of various columns containing mixed media of CHF or PPF in soil system demonstrated that both substrate could also be used as barriers in the landfill to maximize immobilization of toxic metal such as $\mathrm{Cr}(\mathrm{VI})$ in leachates.
\end{abstract}

Keyword: Batch; Column; BDST; Fixed and mixed beds 